\title{
Review Richness: How Online Consumer Review Information Content Shapes Persuasion Through Review Richness: An Abstract
}

\author{
Yiru Wang and César Zamudio
}

\begin{abstract}
Online reviews are a critical electronic word-of-mouth source: firms with helpful reviews are thought of as providing better value, and consumers trust and use reviews to make purchase decisions. Accordingly, research has explored how review features, such as length and valence, influence how persuasive a review is. However, an implicit assumption in the literature is that review length can be used to proxy for how much information is in a review. Yet, two reviews of equal length may contain different amounts of information. To relax this assumption, we conceptualize and propose a new measure of review information content termed "review richness" and an ancillary review complexity measure, constructed based on Shannon's entropy. These measures rely on constructing a lexicon that represents the distribution of words consumers most often use in a category, which reveals words that are often repeated (and thus carry little new content) and words that are more uncommon, providing review readers with more information. Results indicate that review richness is a significant predictor of review helpfulness, particularly for purchases with high expected risk. In terms of predictive ability, adding review richness to a helpfulness model is equivalent to half the predictive ability of review length. Therefore, review richness is a metric that should be included in predictive models of review helpfulness to identify which reviews are most persuasive to review readers.
\end{abstract}

References Available Upon Request

Y. Wang $\bullet$ C. Zamudio $(\bowtie)$

Kent State University, Kent, OH, USA

e-mail: ywang115@kent.edu; czamudi1@kent.edu 